\title{
REGION-BASED IMAGE FUSION USING A COMBINATORY CHEBYSHEV-ICA METHOD
}

\author{
Zaid Omar, Nikolaos Mitianoudis and Tania Stathaki \\ Communications and Signal Processing Group, \\ Imperial College London, \\ South Kensington, \\ SW7 2AZ United Kingdom
}

\begin{abstract}
The aim of this paper is to provide an algorithm for image fusion which combines the techniques of Chebyshev polynomial (CP) approximation and independent component analysis (ICA), based on the regional information of input images. We present a region-based method that combines the merits of both techniques. It utilises segmentation to identify edges, texture and other important features in the input image and subsequently apply the different fusion methods according to regions. The proposed method exhibits better perceptual performance than individual CP and ICA fusion approaches especially in noise corrupted images.
\end{abstract}

Index Terms - Image and data fusion, Chebyshev polynomials, independent component analysis, region-based fusion.

\section{INTRODUCTION}

In critical tracking and surveillance applications, such as defense systems, decisions pertaining the system actions are very rarely made upon the measurements of a single parameter. Often these decisions are based on the inputs of multiple parameters from different sensors in order to reduce the risk of error. In image processing, the combination of different images is called image fusion.

Multisensor systems consist of different modality sensors, for example infrared, laser and visual, each of which provide data that are different and complementary in nature. In the case where the data in question are images, fusion can be defined as a framework that processes this information in order to construct a better quality composite output image. The advantages of multiple sensors over a single sensor are numerous, such as higher SNR, increased robustness and reliability, improved resolution and reduction in measurement time and costs [4].

Fusion methods, and indeed signal decomposition (since fusion is just one of many extensions of signal decomposition), using Chebyshev polynomials (CP) [5] and independent component analysis (ICA) [1] have been well established in the recent literature. Nevertheless, both methods tend to have their own strong and weak points and as such, a novel approach of combining the best aspects of CP and ICA is proposed.

Our method is called the region-based CP-ICA (RBCI) combinatory fusion. This is essentially done by segmenting the images into active and inactive regions or objects, i.e. distinguishing edges and texture as opposed to constant background and mild texture. Canny edge detection is employed for performing this classification

This research was made possible by the fundings of Universiti Teknologi Malaysia and the UDRC. task. Segmentation creates a mathematical boundary between regions which correspond to different objects within a scene. Fusion is then applied individually on each bounded region. The advantages of this technique are two-fold: pixels are more efficiently processed and analysed if they are treated similarly and not completely independently to other neighbouring pixels in the region. Region-based fusion may therefore help to overcome some drawbacks of pixelbased fusion, such as blurring, susceptibility to noise and misregistration [6]. Secondly, this approach will enable us to perform fusion using a mix of CP and ICA, thus getting the best of both methods.

The paper is divided into four sections. The first introduces the concept of image fusion and its applications. Section two discusses incumbent methods for image fusion, namely ICA and CP, their strengths and limitations. The third section elaborates on the proposed method, including segmentation and the combination of the two algorithms. Finally, section four evaluates the performance of RBCI in comparison with incumbent methods in image fusion.

\section{BACKGROUND STUDY}

\subsection{ICA for image fusion}

ICA is an established technique for identifying underlying statistically independent components in a random data set, and remains one of the most efficient methods for image fusion in terms of performance. This is largely due to the fact that ICA comprises a set of bases that are independent to each other and are closely related to the input signal at hand, having been trained from data of similar content. Hyvarinen et al [7] observed that if the ICA algorithm is utilised to analyse image patches from natural images, the estimated transform will consist of independent, localised components that approximate the primary human visual cortex's (V1) method of scenery analysis and edge detection. The concept is akin to signal decomposition in that a complex signal comprises of a combination of various basic frequencies. In the case of ICA, an image patch can be decomposed into several basis patches as shown below in Figure 1 below.

Numerous studies involving ICA for image fusion have been discussed in the literature. These studies vary in terms of fusion rules

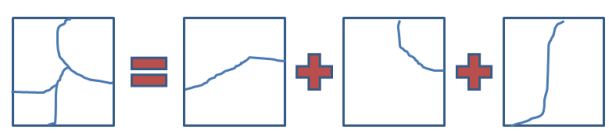

Fig. 1: Image patch decomposed into several basis patches 
used, such as a region-based multimodal images [2, 8] and adaptive ICA [9] to application dependent systems like surveillance in sensor networks [10]. ICA for denoising has also been discussed. ICA is employed to sparsify the image representation, i.e. concentrate most of image's energy into very few components. Then, sparse code shrinkage [11] is employed to filter out weak coefficients in the sparse representation that may be attributed to noise. Instead, CP offer low-frequency approximations of curves and surfaces and they tend to smooth extreme deviations. The difference between sparse code shrinkage and CP's low-pass filtering is that sparse code shrinkage does require a threshold to be calculated beforehand (mainly indicating the level of additive white noise). Instead the CP approximation needs no prior value estimation, but can approximate and fit to any presented waveform or surface.

\subsection{Chebyshev polynomials for image fusion}

Chebyshev polynomials of the first kind, $T_{n}(x)$ are defined as the mathematical solution to the Chebyshev differential equation and can be recursively generated via

$$
T_{0}(x)=1, T_{1}(x)=x, T_{n+1}(x)=2 x T_{n}(x)-T_{n-1}(x) .
$$

The properties of CP have been elaborated in [5]. Chebyshev polynomials can also be used to approximate a signal $f(x)$ with

$$
\tilde{f}(x)=\sum_{n=0}^{N} a_{n} T_{n}(x)
$$

where $\tilde{f}(x)$ is the approximation and $a_{n}$ is a coefficient of $n$ defined by

$$
a_{n}=\frac{2}{\pi} \sum_{x=-1}^{1}(1-x)^{-\frac{1}{2}} f(x) T_{n}(x)
$$

$\mathrm{CP}$ analysis is a form of transformation, where the necessary features are extracted to preserve the second-order image statistics. A finite CP expansion (finite order $n$ ), ensures that only the prominent information are retained, while any redundant statistics are discarded. Conversely, a higher order approximation is more precise, though in the case of corrupted signals noise components tend to occupy the high frequency spectra.

In image processing, the idea is thus to reduce the $\mathrm{CP}$ order in order to remove noise components, effectively acting as a low-pass filter to smooth out noise at a cost of signal accuracy. One advantage is that CP's are derived analytically (as opposed to ICA bases) and thus can be pre-computed and stored in order to reduce processing complexity.

For simple evaluation purposes, the fused image approximation is formed by fusing the two coefficients via the max-abs fusion operator, i.e. choose the coefficient with the higher absolute value. As each coefficient corresponds to the global image intensity and strength, performing max-abs over the two images will retain and enhance the strong pixels while suppressing the weak ones. This is essentially image fusion, resulting in an improved quality image.

Aside from fusion, Chebyshev approximation (sometimes referred to as Chebyshev moments) has been employed for image analysis in the past, mainly for pattern recognition and image segmentation [3], image reconstruction and rendering [12]. Moreover, in [13] the use of Chebyshev approximation has been extended to the estimation of financial data.

\section{THE PROPOSED METHOD}

\subsection{Region-based fusion}

The process of selecting important features from the input images is referred to as fusion, where salient features are extracted in the transform domain so as to provide a better analysis of the image. The outline of the transform domain-based fusion is as follows:

$$
I_{f}(x, y)=\tau^{-1}\left\{g\left(\tau\left\{I_{1}(x, y)\right\}, \ldots, \tau\left\{I_{T}(x, y)\right\}\right)\right\}
$$

where $I_{f}(x, y)$ is the fused image and $I_{1}(x, y), \ldots, I_{T}(x, y)$ are the input images, $\tau(\cdot)$ is the transform operator (in this case Chebyshev approximation and ICA respectively) and $g(\cdot)$ is the fusion rule operator.

The fusion rule lays the guideline for the fusion step at its most basic level. This include pixel-based rules, whereby the coefficient pixels of input images are processed on a general level. In contrast, region-based rules is able to exploit more efficiently the structural information in images. In these schemes, pixels are segmented into larger regions based on criteria such as objects of interest. We can then determine the contribution of regions from each input. Numerous concepts with regards to region-based fusion have been proposed. Among these, [2, 6, 8] are notable examples. As shown later, this is useful particularly in the case of noise corrupted images as noisy regions can be identified and separated during the preprocessing stage.

\subsection{Segmentation algorithm}

Segmentation is defined by considering active and inactive regions [2]. A region is deemed active if there are sufficient "interesting" information within, i.e. edges or complex texture. Inactive regions, on the other hand, imply a 'plain' area, possibly monotonous or regions with very little textural details. In corrupted images, the presence of Gaussian noise tends to pervade the whole image causing an increase in the overall activity scale.

The concept of our approach is that noise is more distinguishable in inactive regions compared to textured regions. Due to the heightened activity in edge-related areas, the presence of any noise may be compromised by textural details within the region and it would therefore be extremely difficult to filter out noise without affecting the regional texture.

ICA is able to capture salient image features more comprehensively and is therefore very efficient at modelling edges and textured regions. On the other hand, the smooth property of Chebyshev polynomials enables them to filter noisy signals better and as such, would be ideal to use on inactive regions mentioned above. In order to reduce computational complexity, noise filtering may be omitted from ICA. Therefore in an image example where edge presence is minimal, we may use CP on most regions while ICA is reserved for scarce edge parts so as to universally preserve signal quality.

Take for example two input images from UN Camp as seen in Figure 2. The images are processed by the Canny edge detection method. The Canny detector is robust to external noise and is essentially a multistage segmentation algorithm that identifies important edges at various scales. The resulting segmentation map for the respective input images are combined via the logical $O R$ operator to form a global segmentation map. The aim of this step is not to accurately determine edges and regions from both inputs, but rather ensure that all important edge details have been preserved in the final segmentation map. 


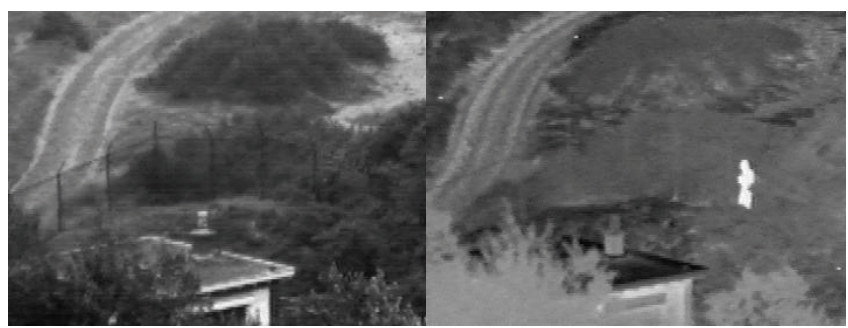

(a) UN1

(b) UN2

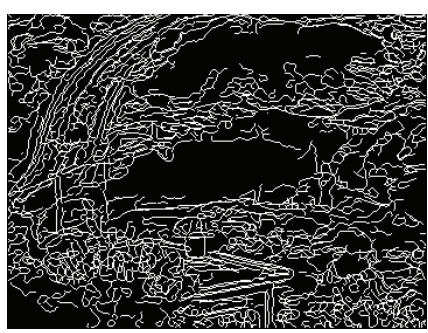

(c) Segmentation map

Fig. 2: Segmentation map derived from selecting all hard and soft edges from both input images

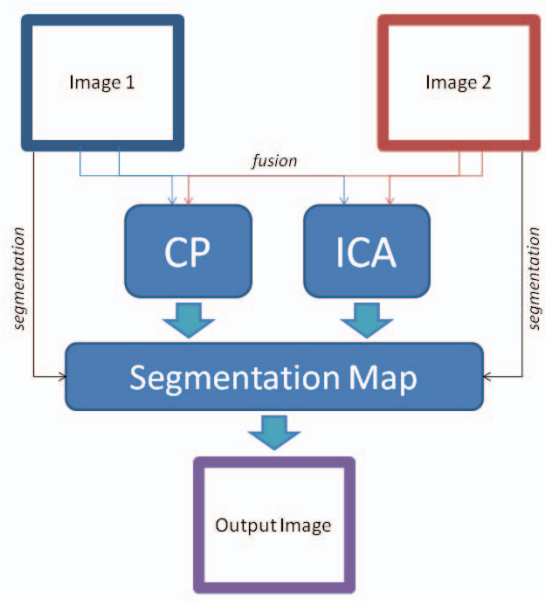

Fig. 3: Framework of region-based combinatory CP-ICA fusion

This map provides a guide of the areas to be processed by either ICA or CP. Active regions represented by white pixels (denoting all hard edges and texture) in the segmentation map as shown in Figure $2 \mathrm{c}$ will be fused using ICA whereas inactive regions via $\mathrm{CP}$. As illustrated in the map, inactive areas form the majority of the image space. Active pixels are limited to object edges and to an extent, texture. This is similar to the way humans generally perceive objects of interest within our line of vision. Background objects are normally plain and tend to carry little information, and as such can be modified, compressed or even omitted. The appeal of this method is it enhances strong edges and texture, thus giving priority to important regions whilst de-prioritising (via smooth approximation of $\mathrm{CP}$ ) non-important background regions.

A generic framework for our method is as in Figure 3.

\section{PERFORMANCE EVALUATION}

The proposed method was tested in multimodal and noisy scenarios respectively. A series of two grayscale images are used as inputs. For the purpose of benchmarking, the ICA-based fusion and Chebyshev fusion methods using the max-abs rule [2] are used as comparisons to RBCI. A $7 \times 7$ frame was used for the ICA bases, whilst $m=11$ and $n=11$ degree of polynomials were used for the $\mathrm{CP}$ and the input image was divided into $7 \times 7$ overlapping patches/windows.

By definition our method does not introduce a new fusion algorithm in itself, but rather is a modified framework from CP and ICA that combines the best aspect of both algorithms. This is seen in Figure 3. The actual fusion part still requires the application of $\mathrm{CP}$ and ICA, and subsequently the respective results are reconstructed together under the combinatory framework.

Computation-wise the RBCI method is relatively heavier than $\mathrm{CP}$ and ICA, owing to the required estimation of both methods coupled with the segmentation and comparison steps involved. However this is redeemed by the method's superior performance in a multitude of scenarios as shown in the results in Figure 4.

The first set of examples tackles the issue of occlusion or obstruction within images. This occurs when two images of the same scene are captured but contain objects occluded in different parts, in this case clouds shrouding a plane in Figures $4 \mathrm{a}$ and $4 \mathrm{~b}$. In Figure $4 \mathrm{~d}$ $\mathrm{CP}$ manages to completely remove the cloud, but produces a blurred image due to its smoothing property. Conversely, in Figure 4c ICA provides a sharp, clear image such that the plane's model number is easily discernible. The drawback though is it did not entirely erase the cloud particles. In this case RBCI in Figure 4e is able to merge the merits of both techniques - it results in a higher quality fusion that completely eliminates unwanted cloud components whilst better preserving the correct objects. The mountain plains in the backdrop has been fittingly smoothed out as it is considered non-important background texture.

Figures $4 \mathrm{f}$ and $4 \mathrm{~g}$ in turn deals with multimodal scenarios whereby an image is captured by sensors of various modalities. The rationale, as stated earlier, is that these sensors are able to provide diverse and complementary information regarding the scene. In this case the aim is concealed weapon detection. In Figure $4 i$ the CP fusion returns an overly smooth image with some loss of important details. ICA's output in Figure 4h on the other hand tends to be grainy (noisy). A higher quality fusion is achieved by RBCI in Figure $4 \mathrm{j}$ which produces a smooth image, purges non-important background details but retains sharp edges from the ICA step.

For noisy image examples, it was noted that $\mathrm{CP}$ approximates the image better due to its smooth property, especially in situations with high level of noise as explored in [5]. However ICA still retains sharp edges and texture and as such is useful in scenarios where noise is minimal. Again, RBCI attempts to integrate both aspects.

To preserve edge details, a dilation step is added to the segmentation map in order to incorporate more pixels from ICA. Furthermore, the $O R$ operator from segmentation is replaced with $A N D$. This ensures that only edges present in both images will be considered for the segmentation map, which may prove to be feasible should only one input becomes corrupted. As edge regions equate to using ICA, this maneuver maximises the use of $\mathrm{CP}$ to suppress noise and limits the use of ICA only to edge lines and hard textural regions which represent important features in an image. The benefit of RBCI is it provides an autonomous denoising capability, combining the benefits of both CP and ICA. 


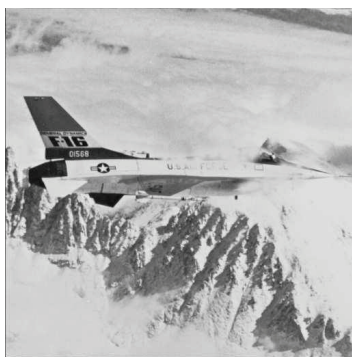

(a) Jet 1

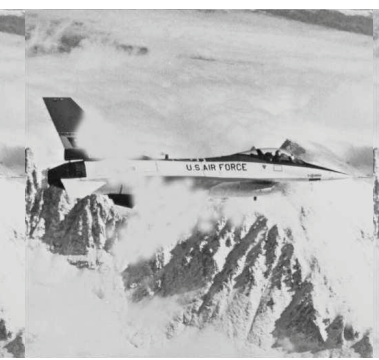

(b) Jet 2

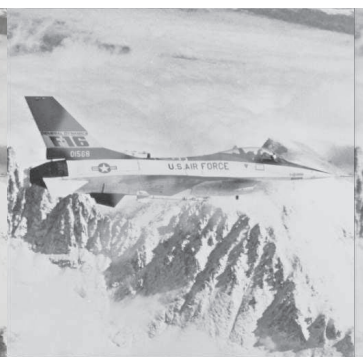

(c) ICA

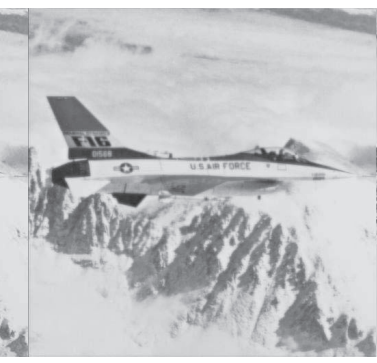

(d) $\mathrm{CP}$

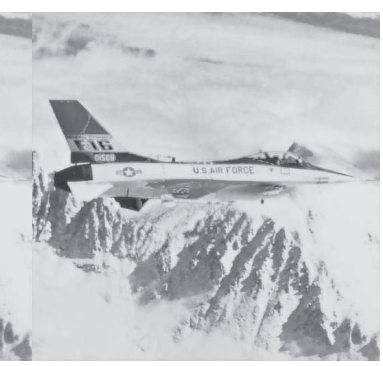

(e) RBCI

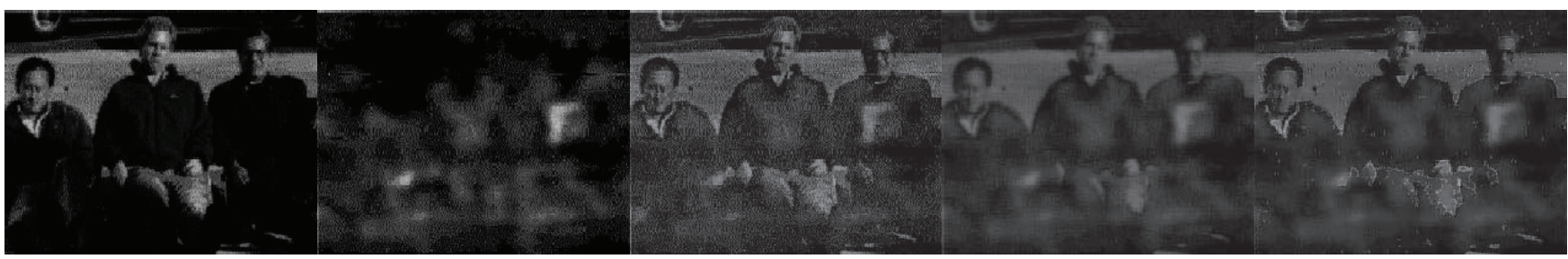

(f) Gun 1

(g) Gun 2

(h) ICA

(i) $\mathrm{CP}$

(j) RBCI
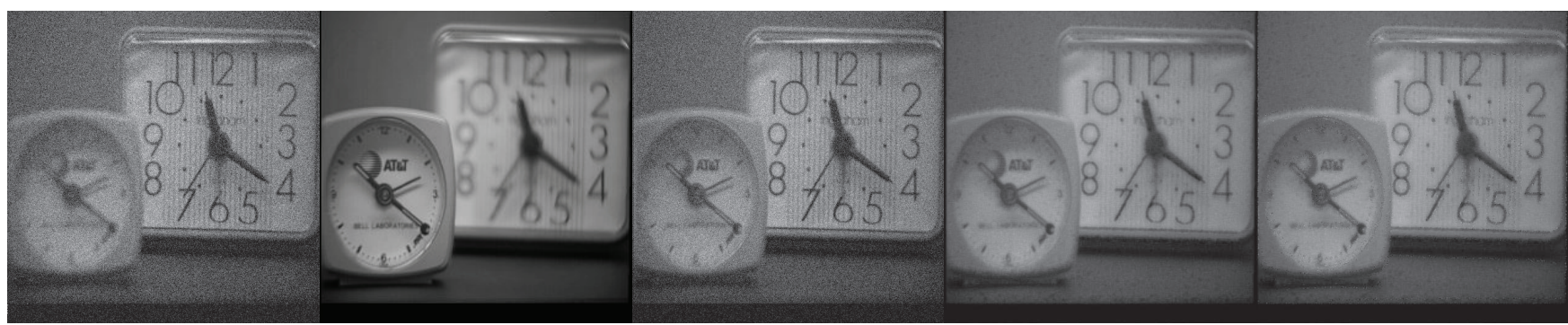

(k) Clock $1(S N R=126.6)$

(1) Clock 2

(m) ICA

(n) $\mathrm{CP}$

(o) RBCI

Fig. 4: Fusion performance of region-based CP-ICA with incumbent methods

\section{REFERENCES}

[1] T. Stathaki, "Image Fusion: Algorithms and Applications", Edited Book, Academic Press, pages 500, 2008.

[2] N. Mitianoudis and T. Stathaki, "Pixel-based and Region-based Image Fusion schemes using ICA bases", Information Fusion, Vol. 8, No. 2, pp. 131-142, 2007.

[3] M. Cheong and K.S. Loke, "Textile recognition using Tchebichef moments of co-occurrence matrices", Advanced Intelligent Computing Theories and Applications. With Aspects of Theoretical and Methodological Issues, Springer Berlin / Heidelberg, Volume 5226/2008, pp. 1017-1024, September 2008

[4] J. Esteban, A. Starr, R. Willetts, P. Hannah and P. Bryanston-Cross, "A review of data fusion models and architecture: towards engineering guidelines, Neural Computing \& Applications, 14: 273-281, SpringerVerlag London Ltd, 2005.

[5] Z. Omar, N. Mitianoudis and T. Stathaki, "Two-dimensional Chebyshev polynomials for image fusion”, 28th Picture Coding Symposium 2010, Nagoya, December 2010.

[6] G. Piella, "A Region-based Multiresolution Image Fusion Algorithm", ISIF Fusion Conference 2002, Annapolis, 2002.

[7] A. Hyvrinen, P. Hoyer and E. Oja., "Image Denoising by Sparse Code Shrinkage". In S. Haykin and B. Kosko (eds), Intelligent Signal Processing, IEEE Press, 2001.
[8] N. Cvejic, J. Lewis, D. Bull and N. Canagarajah, "region-based multimodal image fusion using ICA bases", International Conference on Image Processing 2006, pp. 1801-1804, Atlanta, 2006.

[9] N. Mitianoudis and T. Stathaki, "Adaptive image fusion using ICA bases", IEEE International Conference on Acoustics, Speech, and Signal Processing (ICASSP) 2006, Toulouse, France.

[10] N. Cvejic, D. Bull, N. Canagarajah, "Improving fusion of surveillance images in sensor networks using independent component analysis", IEEE Trans. Consumer Electron., vol. 53, no. 3, pp. 10291035 , 2007.

[11] E. Oja, A. Hyvarinen and P. Hoyer, "Image Feature Extraction and Denoising by Sparse Coding", Pattern Analysis \& Applications (1999) 2: 104-110, 1999

[12] R. Mukundan, S.H. Ong and P.A. Lee, "Image analysis by Tchebichef moments", IEEE Transactions on Image Processing, Vol. 10 No. 9, pp. 1357-1364, 2001.

[13] H. Korizis, "Signal processing methods for the modelling and prediction of financial data", PhD thesis, Communications and Signal Processing Group, Department of Electrical and Electronic Engineering, Imperial College London, January 2008 